\title{
Nitrous oxide oxygen inhalation conscious sedation- A new ambit
}

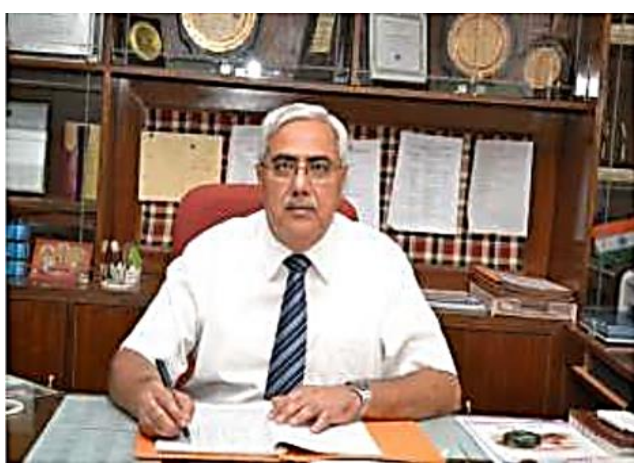

Prof. (Dr.) Vinod Sachdev

Director-PG Studies, I.T.S Centre for Dental Studies and Research, Muradnagar Editor-in-chief

Dear Readers!

Greetings and welcome to the latest edition!

Clinical dental practice frequently witnesses many anxious and skeptical patients specially children. Number of non pharmacological and pharmacological ways to control anxiety have been advocated in such patients. For patients who don't advantage from nonpharmacological behavior modification, Conscious sedation has been the alternative modality. It was kept dormant due to taboos associated with its use in the past but is being used now increasingly in routine dental practice specially for pediatric patients. ${ }^{1}$ Various drugs have been used with different administration routes for conscious sedation, but each one has their own risk index. ${ }^{1}$ Before using and advising any such modality, a trained person with proper protocol is mandatory for any minor or major dental procedure. ${ }^{1}$ A comparatively newer and safer mode for conscious sedation being used by General and Pediatric dentists all over the world is Nitrous Oxide-Oxygen Inhalation Conscious Sedation. ${ }^{2}$ It makes use of mixture of two gases, Nitrous Oxide and Oxygen administered through inhalational route with incremental increase of dosage according to the achievement of desired sedation level subjective to each patient. ${ }^{2}$ One major advantage of this procedure is high safety mechanism incorporated into the standardized machines approved by American Dental Association and American Academy of Pediatric Dentistry available in India. ${ }^{3}$ First and foremost, maximum of $70 \%$ Nitrous Oxide can be delivered and a minimum of $30 \%$ Oxygen is inhaled by the patient during the procedure at all times which is higher than the atmospheric concentration of $21 \%$ for oxygen. Secondly, a fail-safe mechanism is incorporated in the machine which makes the machine non-functional on depletion of oxygen supply. ${ }^{3}$ Thirdly, incremental increase of nitrous oxide concentration in the gaseous mixture allows the operator to keep a check on the appropriate percentage required for conscious sedation. ${ }^{3}$ Fourthly, in case of over sedation, the effect can be reversed immediately by increasing the oxygen concentration as the exchange rate of gases through inhalational route is faster as compared to other routes. ${ }^{3}$ Although, the incorporated safety standard of the approved machine is high, it should only be used under a dental practitioner trained in Basic Life Support., ${ }^{4,5}$ Also, the above mentioned safety mechanisms have reduced and even negated the need of an anesthetist during the procedure. ${ }^{4,5}$ The literature suggests the use of Nitrous Oxide-Oxygen Inhalation Conscious Sedation is completely safe by trained dental practitioners and no untoward event has been reported in the past when used purely in isolation from any other sedative. Dental professional wanting to practice Nitrous Oxide-Oxygen Inhalation Conscious Sedation should be trained under a routinely practicing trained dental practitioner for the same. ${ }^{1,3}$

With the increasing levels of modernization in the dental machinery and their incorporation into the routine dental practice, it is more important for a good institution to harbor all the latest technologies required for providing high quality education and patient care. The Dental Council of India Regulations 2017 recommend the use of Conscious Sedation in all the Colleges providing Master in Dental Surgery (MDS) for the branch of Pediatric \& Preventive Dentistry. Catering to the need of the hour, Nitrous Oxide-Oxygen Inhalation Conscious Sedation has been put to routine use in our institute, I.T.S. Centre for Dental Studies and Research, Muradnagar, Ghaziabad in the Department of Pediatric \& Preventive Dentistry at graduate and post graduate level for providing highest quality of treatment to our patients. Many research projects are ongoing regarding this subject in our Department which suggests that maximum number of pediatric patients get sedation at $40-50 \%$ Nitrous oxide concentration and 
show increased oxygen saturation as compared to baseline with no change in heart rate and respiratory rate. These results are in accordance with the American Academy of Pediatric Dentistry guidelines suggesting $50 \%$ nitrous oxide concentration to be optimal for most pediatric patients. After witnessing the advantages of Nitrous Oxide-Oxygen Inhalation Conscious Sedation in day to day practice, I strongly recommend its use to be made mandatory for routine dental practice especially for Pediatric patients.

I am very thankful to our readers for showing faith in us and making us better with each subscription. I wish you all an incredible reading experience. Please feel free to reach out to us as "Your feedback is important to us".

\section{Regards}

Prof. (Dr.) Vinod Sachdev

Editor-in-chief

Journal of Dental Specialties

\section{References}

1. Guideline for the use of sedation and general anesthesia by dentists.American Dental Association. 2007:1-13.

2. Mohan R, Asir VD, Shanmugapriyan, Ebenezr V, Dakir A, Balakrishnan, Jacob J. Nitrousoxide as a conscious sedative in minor oral surgical procedure. $J$ Pharm Bioallied Sci 2015 Apr; 7(Suppl1): S248-250.

3. Guideline on use of nitrous oxide for pediatric dental patients. American Academy of Pediatric Dentistry. Reference manual. 2013;37:206-10.

4. Paterson SA, Tahmassebi JF. Pediatric dentistry in the new millennium: Use of inhalation sedation in pediatric dentistry. Dent Update 2003;30(7):350-6.

5. Levering NJ, Welie JVM. Current status of nitrous oxide as a behavior management practice routine in pediatric dentistry. J Dent Child 2011;78(1):24-30.

How to cite the article: Sachdev V. Nitrous oxide oxygen inhalation conscious sedation-A new ambit. J Dent Specialities 2018;6(2):90-91. 